\title{
Ten years differences in recently onset atrial fibrillation and flutter incidence and management
}

\section{Differenze a distanza di 10 anni nell'incidenza e nella gestione della fibrillazione atriale di nuova insorgenza}

\author{
Roberto Cemin, Massimiliano Manfrin, Massimo Daves ${ }^{1}$, \\ Werner Rauhe, Aldo Pietro Maggioni2
}

\begin{abstract}
Ten years differences in recently onset atrial fibrillation and flutter incidence and management. R. Cemin, M. Manfrin, M. Daves, W. Rauhe, A.P. Maggioni.

Objects and background: Atrial fibrillation (AF) and atrial flutter (AFl) are the most common arrhythmias in day-life clinical practice. Purpose of our study was to verify differences occurred in the last ten years in AF and AFI incidence and treatment in the emergency room (ER).

Methods: from the 17th January to the 15th February 2000 and from the $18^{\text {th }}$ January to the $16^{\text {th }}$ February 2010 all the consecutive patients with AF or AFI referring to the ER of our hospital were included in the study. Epidemiological data were collected along with information about treatment, admission to hospital wards, days of hospital stay and therapy. Data from the year $\mathbf{2 0 0 0}$ were compared to these collected ten years later.
\end{abstract}

Results: incidence of $\mathrm{AF}$ and $\mathrm{AFl}$ has increased in the years $(50 \%)$, patients are older ( 73.5 vs. 65.2 years; $p$ 0.029) and refer late to the ER $(45.6 \%$ in 2010 and $23.7 \%$ in 2000 with a delay of $>48$ hours from arrhythmias onset; $p 0.054$ ). In 2010 only a minority of these patients is directly discharged from the ER (15.8\% vs $14.4 \%)$ and there is an increased admission rate due to AF or AFl $(67.5 \%$; p 0.026), if compared to the whole admissions of the hospital. The median duration of hospital stay decreased from 6 days to 4.5 days in the year 2010 (NS).

Conclusion: AF and AFl incidence is still increasing and account for a high admission rate from the ER to the hospital wards. Costs are consequently continuously increasing.

Keywords: atrial fibrillation, atrial flutter, emergency room.

Monaldi Arch Chest Dis 2014; 82: 153-159.

Department of Cardiology, San Maurizio Regional Hospital, Bolzano 39100, Italy.

I Clinical Biochemical Laboratory, F.Tappeiner Hospital, Merano 39012, Italy.

2 ANMCO Research Center, Firenze 50100, Italy.

Corresponding author: Dr. Manfrin Massimiliano; Department of Cardiology, San Maurizio Regional Hospital of Bolzano; Via L. Boehler 5; I-39100 Bolzano, Italy; Fax number: 00390471 909977; Telephone number: 00390471 909950; E-mail address: massimiliano.manfrin@sabes.it

\section{Introduction}

Atrial fibrillation (AF) is the most common arrhythmia encountered in day life clinical practice and requires a big human, logistic and economical effort for its correct management. Epidemiological data reports about a $0.4 \%$ prevalence of this rhythm disturbance in general population [1], with an exponential increase in older ages. It is rare under the age of 50, but reaches a prevalence of 5\% at 75 and over $10 \%$ in people older than 80 years [2]. In a minority of patients ranging from 3 to $11 \%$ it could be lone, i.e. not associated to any overt cardiac or extra cardiac disease [3]. More frequently it is related to cardiovascular abnormalities such as congenital, valve or ischemic heart diseases, cardiomiopathy, hypertension or extra cardiac problems like lung disease, electrolytic imbalance, thyroid dysfunction or alcohol abuse. AF usually relapses during life and relapses become more frequent and long lasting after the first episodes.

Atrial flutter (AFl) on the contrary, is not so common and is usually related to mitral valve prob- lems, congenital heart disease, cardiomyopathies, atrial reentrant pathways or lung problems. Its incidence in general population ranges from 0.4 to $0.7 \%$ and it is often associated with AF, with spontaneous change from one rhythm disorder to the other [4]. If not properly treated both these arrhythmias determine a significant life expectancy reduction, with high morbidity and mortality, especially in older ages [5]. The main risk depends from intraatrial thrombus formation and its possible embolisation. Another cause of life expectancy reduction could lie in the high ventricular main rate and the consequent development of heart failure.

These considerations do not apply to lone AF, which is associated to normal life expectancy [3]. Many studies and new international guidelines published in the last ten years, have changed management of AF and AFl [6-8]. Purpose of our study was to verify differences occurred in the last ten years in $\mathrm{AF}$ and Afl incidence in the emergency room (ER) and if the knowledge changes about these rhythm disorders are actually applied in everyday practice. 


\section{Methods}

In the period lasting from the 17th January to the 15th February 2000 we enrolled all the consecutive patients with AF or AFl referring to the ER of our hospital (San Maurizio Regional Hospital of Bolzano, Italy). In order to be included in our registry $\mathrm{AF}$ or $\mathrm{AFl}$ had to be the main problem for seeking medical assistance. Patients with AF or $A F l$, even of new onset, who came to medical attention for a cause non-directly related to the rhythm disturbance, were excluded. Excluded were also patients with permanent $\mathrm{AF}$ or $\mathrm{AFl}$.

All the epidemiological data were collected, with special care to symptoms, supposed causes and duration of arrhythmia, number of relapses, treatment, admission to hospital wards, days of hospital stay and therapy in hospital and at discharge. This first data collection was conducted as part of the national registry FIRE (Atrial fibrillation and flutter Italian registry; 9). Exactly ten years after, in the same 30 days period, lasting from the 18th January to the 16th February 2010 in order to avoid seasonal differences, the observational analysis was repeated. Baseline data were compared to data collected ten years later. main reason for admittance to the emergency room, whilst in the years 2010 they were 57/3864 (1.5\%; 53/57 with AF and 4/57 with Afl). Ten years after, the absolute increase of patients with AF and AFl in the ER in a 30 days period was 19 patients, which corresponds to a $50 \%$ increment and to a statistical significance of the ratios to medical problems with a p level of 0.043 . Age of patients presenting with $\mathrm{AF}$ of AFl in 2000 was $65.2 \pm 17.8$ years and 21/38 $(55.3 \%)$ were males. Age of patients in 2010 was $73.5 \pm 10.8$ years and $34 / 57(59.6 \%)$ were men. There were no gender differences in incidence of these rhythm abnormalities in the ER over the 10 year (p 0.8), whilst patients were older in 2010 (p 0.029 ). The most frequent underlying pathology was hypertension, both in 2000 and in 2010. Lone $\mathrm{AF}$ was present in 2 patients in $2000(5.3 \%)$ and in $3(5.3 \%)$ in 2010 (p NS). Lone AF was defined according to the European Society of Cardiology guidelines [10] as AF that afflict people younger than 60 years and without clinical or echocardiographic abnormalities. Whilst in 4 patients $(10.5 \%)$ in 2000 and in 4 (7\%) in 2010 (p NS) there was no clinical or echocardiographic abnormalities, but they were older than 60 . Clinical characteristics of the two groups are shown in Table 1.

\section{Statistical analysis}

Data were analysed using Microsoft excel version and the statistical program, Analyse-it for Microsoft Excel, Leeds, UK. Means, medians and standard deviations were calculated for continuous variables. Variables were tested for normal distribution with the Shapiro Wilk W test. Significance of differences and frequency distribution of values between the groups were assessed by the Mann-Whitney test for continuous variables and by the Chi-square test or the $\mathrm{F}$ test for categorical variables. $\mathrm{P}$ values of $<0.05$ were considered statistically significant.

\section{Results}

Patients admitted to the emergency room for any problem over the period of 30 days were 7643 (255/day) in the year 2000, and 7477 (249/day) in the year 2010. Medical problems were diagnosed in $4000 / 7643(52.3 \%)$ patients in the year 2000 (133/day) and in $3864 / 7477(51.7 \%)$ in 2010 (129/day; p NS).

In the 30 days period of the year 2000, 38 over 4000 patients with medical problems (1\%) had AF or AFl (35/38 with $\mathrm{AF}$ and $3 / 38$ with $\mathrm{AFl}$ ) as
Table 1. - Clinical characteristic of the two patients groups. Sometimes causes were associated each other

\begin{tabular}{|c|c|c|c|c|}
\hline & & $\begin{array}{c}2000 \\
(\mathrm{~N}=38)\end{array}$ & $\begin{array}{c}2010 \\
(\mathrm{~N}=57)\end{array}$ & $\mathbf{p}$ \\
\hline Male/female & & $14 / 17$ & $111 / 23$ & NS \\
\hline Age (years) & & $65.2 \pm 17.8$ & $73.5 \pm 10.8$ & 0.0298 \\
\hline HR & & $125(90-160)$ & $121(67-170)$ & NS \\
\hline Diabetes & & $8(21 \%)$ & $15(26.3)$ & NS \\
\hline \multirow[t]{4}{*}{ Previous events } & 0 & $19(50 \%)$ & $25(43.8 \%)$ & NS \\
\hline & $1-2$ & $13(34.2 \%)$ & $11(19.2 \%)$ & NS \\
\hline & $\geq 3$ & $4(10.5 \%)$ & $21(36.8 \%)$ & 16 \\
\hline & Unknown & $2(5.3 \%)$ & $0(0 \%)$ & NS \\
\hline \multirow[t]{8}{*}{ Underlying pathology } & Lone AF & $2(5.3 \%)$ & $3(5.3 \%)$ & NS \\
\hline & Hypertension & $14(36.8 \%)$ & $39(68.4 \%)$ & 0.0047 \\
\hline & Valvular & $4(10.5 \%)$ & $4(7.0 \%)$ & NS \\
\hline & Dilated CMP & $2(5.3 \%)$ & $5(8.8 \%)$ & NS \\
\hline & Ischemic & $8(21.1 \%)$ & $11(19.3 \%)$ & NS \\
\hline & Congenital & $0(0 \%)$ & $0(0 \%)$ & NS \\
\hline & Others & $3(7.9 \%)$ & $6(10.5 \%)$ & NS \\
\hline & Unknown & $6(15.8 \%)$ & $3(15.8 \%)$ & NS \\
\hline
\end{tabular}

Age $>60$ without

other causes

$4(10.5 \%)$

$4(7.0 \%)$ NS

2000: patients enrolled in the year 2000; 2010: patients enrolled in the year 2010; HR: heart rate (data are shown as mediand and $5^{\text {th }}$ to $95^{\text {th }}$ percentiles in brackets); AF: atrial fibrillation; CMP: cardiomyopathy; None: non causes and age $>55$ years. 


\section{Symptoms}

Symptoms were usually depending from the elevate heart rate. Palpitations and sudden onset of dyspnoea were the mostly referred causes of admittance to the emergency room both in 2000 and in 2010. Symptoms did not differ between 2000 and 2010.

\section{Time from rhythm disturbance onset}

Anyway, in $2000 \mathrm{AF}$ or AFl arouse within 48 hours from admission in the ER in 28 patients (73.7\%) and more than 48 hours before in 9 patients $(23.7 \%)$; in 1 patient $(2.6 \%)$ the duration of the arrhythmia remained unknown. In 2010, recent onset of $\mathrm{AF}$ or $\mathrm{AFl}$ (within 48 hours from admission) was present in $31 / 57$ patients $(54.4 \%)$, whilst in 26 (45.6\%) AF or AFl arouse more than 48 hours before. There was no significant difference in time onset of the arrhythmias between the year 2000 and 2010 (p 0.054). Patients with relapse did not refer to the ER earlier than patients with the first event ( $p$ 0.2 ), demonstrating the lack of educational training.

\section{Relapses}

In the year 2000, 19 of the 38 patients referring to the $\mathrm{ER}$ for $\mathrm{AF}$ or $\mathrm{AFl}(50 \%)$, were experiencing their first arrhythmic episode, 13 (34.2\%) had had previously $1-2$ episodes and $4(10.5 \%)$ more than 2 episodes. In two cases this information was missing.

In the year 2010, 25 of the 57 patients referring to the ER for $\mathrm{AF}$ or $\mathrm{AFl}(43.8 \%)$ had the first episode, $11(19.2 \%)$ patients had had 1-2 previous episodes and $21(36.8 \%)$ more than 2 previous episodes.

The percentage of first episodes did not change in the 10 years period ( $p$ 0.7), as did not the percentage of patients with 1-2 previous episodes ( $p$ $0.16)$. On the contrary, patients with more than two previous episodes were significantly more in 2010 (p 0.009).

\section{Treatment in the emergency room}

In the year 2000, over the 38 patients referring to the $\mathrm{ER}$ for $\mathrm{AF}$ and $\mathrm{AFl}, 27(71 \%)$ did not receive any treatment, 7 (18.4\%) were given propafenone intravenously, $2(5.2 \%)$ received digitalis and 2 (5.2\%) underwent transoesophageal atrial stimulation.

In the year 2010, over the 57 patients referring to the ER for AF and AFl, 44 (77.2\%) did not receive any treatment, $7(12.2 \%)$ were given propafenone intravenously, $3(5.2 \%)$ flecainide, 1 (1.7\%) betablockers, $1(1.7 \%)$ underwent transoesophageal atrial stimulation and $1(1.7 \%)$ an electrical cardioversion.

With these small numbers comparison are difficult, but the rate of patients not receiving any treatment in the emergency room is still high and has not decreased in the observed period ( $p 0.66$ ).

Therapy in the ER was almost ever administered to patients with arrhythmias appeared within the previous 48 hours. Among patients directly discharged from the ER (5/38 in the year 2000 and 9/57 in the year 2010), 80\% (4/5) were discharged in sinus rhythm in 2000 and 67\% (6/9) in 2010 (NS).

\section{Admission to hospital wards}

In the year 2000 over the 30 days period of the study, 33/38 patients $(86.8 \%)$ admitted to the ER with $\mathrm{AF}$ or $\mathrm{AFl}$, were hospitalised in a medical ward. The hospitalisation for these 5 problems accounted for the $1.97 \%$ of the total amount of admissions (medical and surgical) in the same period and for $2.98 \%$ of the admissions for a medical problem.

Ten years later, in the same 30 days period, $48 / 57$ patients $(84.2 \%)$ admitted to the ER with AF or AFl were hospitalised in an internist ward. The hospitalisation for these problems accounted for the $3.3 \%$ of the total amount of admissions (medical and surgical) in the same period and for the $4.75 \%$ of the admissions for medical problems.

The percentage of hospitalisation due to $\mathrm{AF}$ and $\mathrm{AFl}$ seems very high. Admissions for these arrhythmias over the total amount of hospitalisation in the 30 days period has increased in the comparison year 2000-2010: increase of $67.5 \%$ if compared to total hospitalisation (surgical and medical) and increase about $59.4 \%$, if compared to hospitalisation only for medical problems ( $\mathrm{p} 0.026$ and $\mathrm{p}$ 0.11 respectively).

In the year 2000, the hospital wards in which patients were admitted were Cardiology in 4/33 patients $(12.1 \%)$, Internal Medicine in 22/33 patients $(66.7 \%)$ and other wards (Geriatrics, Short stay ward) in $7 / 33$ patients $(21.2 \%)$.

Ten years later the wards of admission were Cardiology in 4/48 patients (8.2\%), Internal Medicine in $35 / 48$ patients $(74.5 \%)$ and other wards (Geriatrics, Short stay ward) in $9 / 48$ patients $(19.2 \%)$. The wards of admission did not change in percentage

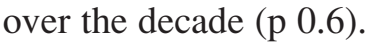

\section{Examinations and treatment during admission}

Electrical cardioversion for patients admitted from the emergency room was rare in the year 2000 (4/33 patients; $12 \%)$ and is still rare ten years after (7/48 patients; $14.6 \%$ ). There has been no difference over the decade in the use of this method for restoring sinus rhythm (p NS). Even the pharmacological conversion to sinus rhythm is low $(9 / 33 \mathrm{pa}-$ tients; $27.3 \%$ in 2000 and $12 / 48$ patients; $25 \%$; p NS). Consequently sinus rhythm restore is relatively low (21/33 patients; $63.6 \%$ in 2000 and 29/48; $60.4 \%$ patients in 2010; NS).

Sub analysis of different treatment received in different wards, although interesting, was not conducted because of the small number of the patients included.

Fast strategy to restore sinus rhythm after performing a transoesophageal echocardiography accordingly to the ACUTE protocol [11], was almost never used both in the year 2000 and in the year 2010 (1/33 and $3 / 48$ patients in 2010 respectively; $p$ NS). The rate of electrical cardioversion or pharmacological cardioversion in all the patients (directly discharged from the emergency room and admitted to the wards) is shown in Table 2.

Concerning drug therapy, during hospital admission major antiarrhythmics (propafenone, flecainide, amiodarone or sotalol) were used in 16/33 patients in $2000(48 \%)$ and in $19 / 48(39.5 \%)$ in 2010 (p 0.57). Therapy for rate control changed over the 
Table 2. - Electrical cardioversion or pharmacological conversion to sinus rhythm in all the patients (directly discharged from the emergency room and admitted to the wards)

\begin{tabular}{lccc}
\hline & $\begin{array}{c}2000 \\
(\mathrm{~N}=38)\end{array}$ & $\begin{array}{c}2010 \\
(\mathrm{~N}=57)\end{array}$ & $\mathrm{p}$ \\
\hline ECV & $4(10.5 \%)$ & $7(12.3 \%)$ & $\mathrm{NS}$ \\
\hline PCV & $9(23.7 \%)$ & $12(21.1 \%)$ & NS \\
\hline
\end{tabular}

2000: patients enrolled in the year 2000; 2010: patients enrolled in the year 2010;

ECV: electrical cardioversion;

PCV: pharmacological conversion.

decade: in 2000 betablockers were used only in $1 / 33$ patients (3\%), whilst in 2010 they were administered in $15 / 48$ (31.2\%); p 0.004. In the year 2000 there was almost the same use of verapamil/diltiazem $(8 / 33$ patients; $24.2 \%$ vs. $9 / 48 ; 18.7 \%)$, whilst digitalis was much more prescribed $(20 / 33 ; 60.6 \%$ vs. $7 / 48 ; 14.5 \%$ ) if compared to 2010 (p 0.75 and $<0.0001$ respectively).

Concerning the other main topic of AF, i.e. oral anticoagulant or antiplatelets therapy, there was an increase in oral anticoagulation prescription. In the year 2000 anticoagulation (heparin or Low Molecular Weight Heparin and oral anticoagulation or a combination of both) was performed in $19 / 33$ patients admitted to the wards $(57.6 \%)$. In 13 of the 14 patients not treated with anticoagulant therapy, received antiplateles agents $(39.4 \%$ of the whole). In 2010 anticoagulation was performed in $30 / 48$ patients admitted to the wards $(62.5 \%)$. Of the 18 patients not treated with anticoagulant therapy, 16 received antiplateles agents $(33.3 \%$ of the whole).

Differences between patients treated with anticoagulant therapy were not significant in the comparison year 2000/year 2010 (p 0.63) and not significant were also differences in antiplateles prescription (p 0.75).

Echocardiography, which should be part of the standard work up of these patients, was performed in $15 / 33$ patients $(45.5 \%)$ in 2000 and in $14 / 48$ patients (29.2) in 2010; p NS.

\section{Duration and costs of hospital admittance}

The total duration of hospital stay for the $33 \mathrm{pa}$ tients hospitalised in the year 2000, was 241 days (median 6 days; 5-95 percentile: 1-30.4 days) and 285 days in the year 2010 (median 4.5 days; 5-95 percentile: 1-17.6 days). Although there was a trend towards a shorter hospital stay in 2010, the different duration of hospital admission was not statistically

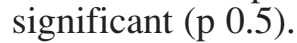

Mean cost per day of admittance in a non intensive ward of our hospital is about 274 Euros and rises to about 820 Euros for the cardiac intensive care unit. The total cost of admittances for the $47 \mathrm{pa}-$ tients with recent onset of AF or AFl, who were hospitalised in 2010 was 81366 Euros. In these costs specialist examinations like echocardiography, $\mathrm{X}$ rays and laboratory tests were not included.

\section{Hospital discharge}

Over the 33 patients admitted in the year 2000, 31 were discharged at home, whilst $2(6.1 \%)$ died because of causes not related to AF or AFl.

Twenty over thirty-one patients $(64.5 \%)$ discharged alive from the hospital wards in the year 2000 were in sinus rhythm at discharge. In 7/11 patients discharged in $\mathrm{AF}$, an electrical cardioversion was planned.

In the year 2010 results were similar. Over the 48 patients admitted, 45 were discharged at home, whilst $3(6.2 \%)$ died because of causes non related to AF or AFl. Sinus rhythm was present in $28 / 45 \mathrm{pa}-$ tients discharged alive $(62.2 \%)$. In $3 / 17$ patients discharged in AF, an electrical cardioversion was planned.

The percentage of sinus rhythm restore in patients admitted to the wards remained almost stable in the ten years interval ( $p 0.9)$, whilst the number of programmed electrical cardioversion in patients discharged in AF decreased significantly ( $p$ 0.038).

In the year 2000 therapy at discharge included a major antiarrhythmic agent for rhythm control in $14 / 31$ patients $(45.2 \%)$. Heart rate control agents consisted of betablockers in $1 / 31$ patients $(3.2 \%)$, verapamil/diltiazem in $3 / 31(9.7 \%)$ and digitalis in $12 / 31(38.8 \%)$ Oral anticoagulant therapy was prescribed in $9 / 31$ patients $(29.0 \%)$ and antiplatelets agents in $11 / 31(35.5 \%)$.

Ten years later therapy at discharge included major antiarrhythmic agent in 16/45 patients (35.6\%; p 0.5 vs. the year 2000), betablockers in $14 / 45$ (31.1\%; p 0.007 vs. the year 2000), verapamil/diltiazem in $8 / 45(17.8 \%$; p 0.5 vs. the year $2000)$ and digitalis in $6 / 45$ patients $(13.3 \%$; 0.022 vs. the year 2000).

Oral anticoagulation at discharge was prescribed in $26 / 45$ patients $(57.8 \%$; $\mathrm{p} 0.025$ vs. the year 2000$)$ and antiaggregation in $14 / 45$ (31.1\%; NS vs. the year 2000).

A further analysis about congruence of indication and actual prescription of oral anticoagulation or antiaggregation at discharged was performed. Thought guidelines for anticoagulation have changed in the last years the congruence was not analysed for the year 2000 .

Mean CHADS2 score [13] in 2010 resulted 1.85 , with a standard deviation of 0.91 . We considered a CHADS2 score of 1 as an indication to aspirin and of $\geq 2$ as an indication to oral anticoagulation. If compared to the CHADS2 score, globally there was an inadequate prescription in $17 / 45$ patients (37.8\%). 8 patients had an indication to oral anticoagulation and were discharged only on aspirin, 1 had an indication to oral anticoagulation and was discharged without anticoagulant and antiplatelets therapy, 2 had an indication to aspirin and were discharged without it. On the other side, there was an over prescription of oral anticoagulant in 6 patients with a CHADS2 score of only 1 .

Recent guidelines on AF management [14] suggest to consider risk factors for thromboembolism slightly differently from the CHADS2 score and accept aspirin or oral anticoagulation in patients with one moderate risk factor, depending from patient's choice. 
Therefore, following guidelines, inadequate prescription of anticoagulant/antiaggregant therapy was found in $13 / 45$ patients $(28.9 \%)$, being the over prescription of oral anticoagulant reduced to 2 patients. Other 2 patients had indications to aspirin or oral anticoagulant and were discharged without them, 1 had indication to aspirin and did not receive it and 8 patients had indication to oral anticoagulation and were discharged only on aspirin.

Considering guidelines or CHADS2 score, there was the same percentage of wrong prescription of aspirin instead of oral anticoagulation, which accounted for $17.8 \%$ of the cases.

\section{Discussion}

Our data about the increased incidence of newly developed AF or AFl in the emergency room in the last decade confirms previous hypothesis of a continuous dramatically rise of this rhythm disturbance over years [15]. Many resources are drained from these patients in terms of visits, admissions to hospital, instrumental and laboratory examinations and therapies, which often needs a multidisciplinary approach.

We observed a $50 \%$ increase in the absolute number of patients with $\mathrm{AF}$ or $\mathrm{AFl}$ admitted to the ER over a ten-year period. The percentage of these rhythm disorders over the whole cases of medical problems in the ER raised from 1\% in the year 2000 to $1.5 \%$ in the year 2010 .

Similarly, in the considered period, the admissions to hospital wards from ER because of these arrhythmias increased over the decade in an absolute percentage of $67.5 \%$ if compared to the whole admissions (surgical and medical) and in a percentage of $59.4 \%$ if only admissions because of medical problems were considered.

This fact could lie in the delay of ER access, which contraindicates immediate restore of sinus rhythm. Alternatively it could depend from the need of rapid turnover of the patients in ER due to overworking or to scarcity of doctors and nurses. For the same reasons therapy in the ER was very seldom administered.

Our observations amplify data shown in the US 20 years ago, where admissions because of AF or AFl accounted for $40 \%$ of the admissions because of rhythm disorders, which represented themselves $1.5 \%$ of total hospital stay $[16,17]$.

Patients with these rhythm problems were older and had experienced more relapses in 2010 respect to ten years earlier.

Our observed duration of hospital admission is once again similar to reported data of 5 days of stay collected in the States [17-18]. Hospital stay has decreased over the decade, but not in a significant way, being the median 6 days in 2000 and 4.5 in 2010.

This observation shows how complex and challenging treatment of these problems in real word medicine is. Many protocols, like early electrical cardioversion after excluding the presence of an intracardiac thrombus, have been proposed to reduce hospital stay and improve assistance, but they are scarcely used in clinical routine.
Interesting is the observation that, despite the presence of relevant symptoms like palpitation, dyspnoea, syncope or angina, only a minority of the cases refers to the emergency room in the first 48 hours, which are usually considered the useful time to try to restore the normal sinus rhythm [14] avoiding an increased embolic risk. Only $73.7 \%$ of the patients with these problems sought medical assistance in the first 48 hour in 2000 and this percentage has decreased to a poor $54.4 \%$ in 2010 .

Even if it is not always necessary to accede to the ER immediately after symptoms onset, because the arrhythmia could disappear itself spontaneously, a too late referral reduces the success rate of cardioversion and increases the embolic risk. The high percentage of patients referring to ER after the first 48 hours could suggest the need for a sensibilisation of the population towards these problems, as it has been done for myocardial infarction. Our data point out some further considerations.

The use of electrical cardioversion or pharmacological conversion was very low in 2000 and it remains low now. Consequently the percentage of sinus rhythm restore at hospital discharge is not so high and it has not improved over the decade $(64.5 \%$ to $62.2 \%$ ). This could once more depend on the late arrival to the ER from the first onset of the arrhythmia, but we did not observe any difference in the percentage of cardioversion between patients presenting early or late. Other explanations could be a high number of patients with contraindications to major antiarrhythmics, a high level of co morbidity that advise against an immediate treatment of the problem or a different cultural approach to the rhythm problem. A low aggressiveness in trying to restore sinus rhythm and a higher inclination to transform newly onset $\mathrm{AF}$ in permanent $\mathrm{AF}$ could derive from the results of large clinical studies [6, 7]. This could be also supported by the observation that planned electrical cardioversions in patients discharged on $\mathrm{AF}$ decreased from $63.6 \%$ to $17.6 \%$ in the ten-year period.

Echocardiography, which should be part of the standard work up of these patients, is still underused and was performed in $15 / 33$ patients $(45.5 \%)$ in 2000 and in 14/48 patients (29.2) in 2010; p NS.

In the same period there was also a change in therapy choice at discharge. Major antiarrhythmics were prescribed almost to the same proportion of patients, whilst agents for rate controls showed a dramatically reduction in the use of digitalis and an increased prescription of betablockers. The use of verapamil or diltiazem remained almost unchanged. These observations could suggest a greater adherence to guidelines concerning the strategy of heart rate control in patients with $\mathrm{AF}$.

In the matter of the other main topic of therapy in $\mathrm{AF}$ and $\mathrm{AFl}$, i.e. anticoagulation, data are still disappointing. Considering guidelines or CHADS2 score, in 2010 there was the same percentage of under prescription of warfarin to patients having the indication, than was observed 10 years ago (18\%).

Both in 2000 and in 2010 no death were directly related to the arrhythmia. In these cases AF has precipitated the clinical condition and was the reason for referring to the emergency room. Finally some 
considerations about the costs. The percentage of hospital admission from the ER for these patients is very high and has increased in the decade. The mean duration of hospital admission showed a trend towards a shorter stay in 2010 , as compared to the 2000 , but this difference was not statistically significant. Anyway, the admission period of 4.5 days is still too high, considering the pathology afforded, which could be often treated by a well organised outpatient clinic. Consequently costs remain very high and could be reduced.

\section{Conclusion}

Our study confirms once more that AF and AFl incidence is dramatically increasing in the years [19] and only a minority of these patients is directly discharged from the ER and admissions are usually long lasting. Transoesophageal cardioversion and subsequent electrical cardioversion accordingly to the ACUTE protocol [11] is still almost never used. Even in the patients arriving early, the rate of conversion to sinus rhythm in the emergency room is very low and the global percentage of sinus rhythm restore in patients discharged from the hospital is also low and has not increased over the decade, being $62.2 \%$ in 2010 .

Lessons from the big trial or new international guidelines seem to have had only minor influence in real word treatment of these rhythm disorders.

Considering that the public health burden of atrial fibrillation is enormous and is expected to increase continuously over the next decades, as showed from our observations, integrated protocols should be developed as soon as possible, in order to afford globally and properly this problem.

Furthermore, primary prevention of AF should be performed, treating risk factors like hypertension as soon as possible.

\section{Riassunto}

Premessa ed obiettivo dello studio: La fibrillazione atriale $(F A)$ ed il flutter atriale (FlA) sono le aritmie di più comune riscontro nella pratica clinica quotidiana. Scopo del nostro studio è stato verificare le differenze negli ultimi 10 anni dell' incidenza e del trattamento di FA e FlA in pronto soccorso (PS).

Metodi: Dal 17 gennaio al 15 febbraio del 2000 e dal 18 gennaio al 16 febbraio 2010 sono stati inclusi nello studio tutti i paziente consecutivi che si sono recati nel PS del nostro ospedale per FA o FlA. Oltre ad i dati epidemiologici sono state raccolte informazioni sul trattamento, sui ricoveri nei reparti ospedalieri, sui giorni di degenza e sulla terapia. I dati del 2000 sono poi stati confrontati con quelli raccolti nel 2010.

Risultati: l'incidenza del FA e del FlA sono aumentate in 10 anni (50\%), i pazienti sono più anziani (73.5 vs. 65.2 years; $p$ 0.029) e si recano più tardivamente in PS $145.6 \% \mathrm{nel} 2010$ e $23.7 \% \mathrm{nel}$ 2000 si sono recati in PS più di 48 ore dopo l'insorgenza dell'aritmia; $p$ 0.054). Nel 2010, cosi come nel 2000, solo una minoranza dei pazienti sono stati dimessi direttamente dal PS (15,8\% vs 14,4\%), mentre si è registrato un aumento in percentuale dei ricoveri per FA e FlA comparati al totale dei ricoveri in ospedale. La durata media della degenza è scesa da 6 giorni nel 2000 a 4,5 giorni nel 2010 $(p=N S)$.

Conclusioni: FA e FlA sono in continuo aumento come incidenza e ricoprono una grossa percentuale dei ricoveri in ospedale effettuati dal PS. Di conseguenza i costi sono in continuo aumento.

Parole chiave: fibrillazione atriale, flutter atriale, Pronto Soccorso.

\section{ABBREVIATIONS}

AF: atrial fibrillation

$\mathrm{AFl}$ : atrial flutter

ER: emergency room

\section{Limitation of the study}

- Main shortcoming of the study was the small number of the patients included. This depends on the inclusion criteria and on the purpose of the study, which was intended to be a spot observation comparing incidence and treatment of the same pathology in the same centre after 10 years. Of course this leads sometime to difficulties in statistical comparison.

- Another limit is that it was not easy to record the arrhythmia duration and sometime it remained unknown.

Acknowledgments: The authors are greatly indebted to Dr. Franco De Giorgi for the collaboration.

\section{References}

1. Camm AJ, Lip G, De Caterina R. 2012 focused update of the ESC guidelines for the management of atrial fibrillation; Eur Heart J 2012; 33: 2719-2747.

2. Ryder KM, Benjamin EJ. Epidemiology and significance of atrial fibrillation. Am J Cardiol 1999; 84: 131-138.

3. Jahangir A, Lee V, Friedman PA. Long-term progression and outcomes with aging in patients with lone atrial fibrillation: a 30-year follow-up study. Circulation 2007; 115: 3050-6.

4. Bellet S. Clinical disorders of the heart beat. Philadelphia, Lea \& Febinger, 1963; pg. 144-145.

5. Stewart S, Hart CL, Hole DJ, McMurray JJ. A population-based study of th long-term risks asspcoated with atrial fibrillation: 20-year follow-up of the Renfrew/Paisley study. Am J Med 2002; 113: 359-364.

6. Wyse DG, Waldo AL, DiMarco JP. A comparison of rate control and rhythm control in patients with atrial fibrillation. $N$ Engl J Med 2002; 347: 1825-1833.

7. Hohnloser SH, Kuck KH, Lilienthal J for the PIAF Investigators. Rhythm or rate control in atrial fibrillation Pharmacological Intervention in Atrial Fibrillation (PIAF): a randomised trial. Lancet 2000; 356: 1789-1794.

8. Estes NA 3rd, Halperin JL, Calkins H. American College of Cardiology; American Heart Association Task Force on Performance Measures; Physician Consortium for Performance Improvement. ACC/AHA/Physician Consortium 2008 clinical performance measures for adults with nonvalvular atrial fibrillation or atrial flutter: a report of the American College of Cardiology/American Heart Association Task force on performance measures and the physician consortium for performance improvement (writing committee to develop clinical performacne measures for atrial fibrillation). Developed in collaboration with the Heart Rhythm Society. J Am Coll Cardiol 2008; 51: 865-884. 
9. Santini M, De Ferrari GM, Pandozi C. Atrial fibrillation requiring urgent medical care. Approach and outcome in the various departments of admission. Data from the atrial Fibrillation/flutter Italian REgistry (FIRE). Ital Heart J 2004; 5: 205-213.

10. Vahanian A, Auricchio A, Bax J et al. Guidelines for the management of atrial fibrillation. European Heart Journal 2010; 31: 2369-2429.

11. Asher CR, Klein AL. The ACUTE trial. Transesophageal echocardiography to guide electrical cardioversion in atrial fibrillation. Assessment of Cardioversion Using Transesophageal Echocardiography. Cleve Clin J Med 2002; 69: 713-718.

12. Hart RG, Pearce LA. Current status of stroke risk stratification in patients with atrial fibrillation. Stroke 2009; 40: 2607-2610.

13. Fuster V, Rydén LE, Cannom DS. ACC/AHA/ESC 2006 guidelines for the management of patients with atrial fibrillation Europace. 2006 Sep; 8(9): 651-745.
14. GO AS, Hylek EM, Phillips KA. Prevalence of diagnosed atrial fibrillation in adults: national implications for rhythm management and stroke prevention: the AnTicoagulation and Risk Factors in Atrial Fibrillation (ATRIA) Study. JAMA 2001; 285(18): 2370-2375.

15. Khairallah F, Ezzedine R, Ganz LI, London B, Saba S. Epidemiology and determinants of outcome of admissions for atrial fibrillation in the United States from 1996 to 2001. Am J Cardiol 2004; 94: 500-504.

16. Bialy D, Lehmann MH, Schumacher DN, Steinmann RT, Meissner MD. Hospitalisation for arrhythmias in the United States: importance of atrial fibrillation. J Am Coll Cardiol 1992; 19 (3): 41 A.

17. Geraets Dr, Kienzle MG. Atrial fibrillation and atrial flutter. Clin Pharm 1993; 12: 721-735.

18. Singer DE, Chang Y, Fang MC. The net clinical benefit of warfarin anticoagulation in atrial fibrillation. Ann Intern Med 2009; 151: 297-305. 